\title{
OTIONOMICS
}

Revista de economía, empresa y sociedad

Dosier "Género y empresa: hacia la igualdad real»

Coordinadora: Laura Lamolla Kristiansen

BALANCE Y PROPUESTAS DE MEJORA

\section{La (des)igualdad de género en las organizaciones: avances y estancamientos en España}

\section{Susana González}

Profesora colaboradora del departamento de Economía de la Empresa de la Universidad CEU San Pablo.

\section{Ruth Mateos de Cabo}

Profesora titular del departamento de Economía de la Empresa de la Universidad CEU San Pablo.

RESUMEN En el presente trabajo se analizan los avances en materia de igualdad de género que han contribuido a que España se sitúe entre los 10 primeros países en la última edición del Gender Gap Report del Foro Económico Mundial. Gran parte de dicho avance se debe a la mejoría en la representación política de las mujeres en posiciones ministeriales y en el parlamento. No obstante, la participación económica de las mujeres en España muestra amplios espacios de mejora, especialmente en lo relacionado con la representación en puestos de liderazgo empresarial y con una preocupante infrarrepresentación en las profesiones emergentes fruto de la transformación digital. Para finalizar, el trabajo ofrece una serie de recomendaciones para mejorar el empoderamiento económico de las mujeres a la hora de acceder a posiciones de alta responsabilidad empresarial, así como para prevenir y mejorar en el futuro la brecha económica relacionada con su infrarrepresentación en profesiones relacionadas con la ciencia, la investigación y la tecnología.

PALABRAS CLAVE igualdad de género; cuotas; consejos; STEM; transformación digital 


\title{
Gender (in)equality in organisations: Progress and stagnation in Spain
}

\begin{abstract}
This work analyses the advances in regard to gender equality which have contributed to the fact that Spain ranks among the 10 leading countries in the latest edition of the Gender Gap Report by the World Economic Forum. This progress is mostly due to the improvement in the political representation of women in ministerial positions and in parliament. However, the economic participation of women in Spain evidences extensive room for improvement, especially in relation to the representation in corporate leadership positions and to a worrying underrepresentation in the emerging professions resulting from the digital transformation. To conclude, the work offers a series of recommendations for improving the economic empowerment of women when it comes to reaching positions of high corporate responsibility. It also recommends to prevent and improve the future economic gap in respect to women's underrepresentation in science, research and technology professions.
\end{abstract}

KEYWORDS gender equality; quotas; boards of directors; STEM; digital transformation

\section{Avances en materia de igualdad de género en España}

España es uno de los países que más ha mejorado en la última edición del Global Gender Gap Report (GGGR) elaborado por el Foro Económico Mundial, pasando de ocupar el puesto 29 al puesto 8, de entre 153 países en 2019 (World Economic Forum, 2020), estableciéndose así como líder en la lucha contra la discriminación de género en los ámbitos social, político y económico.

El factor principal que ha contribuido a este repunte de España en el GGGR ha sido el empoderamiento político de las mujeres (figuras 1 y 2). En 2019, España se convirtió en el gobierno con más mujeres del mundo, con un 65 \% de ministras en el gabinete de Pedro Sánchez (11 ministras de un total de 17). Además, el Congreso español se convirtió en el más igualitario de la UE con un 47,4 \% de mujeres (166 diputadas de 350) después de las elecciones de abril del 2019. Esto significó que España superó a países como Suecia y Finlandia. Sin embargo, las elecciones de noviembre, después del bloqueo político en el país, redujeron esta cifra al $43,1 \%$. 
Figura 1. Principales indicadores para España del GGGR.

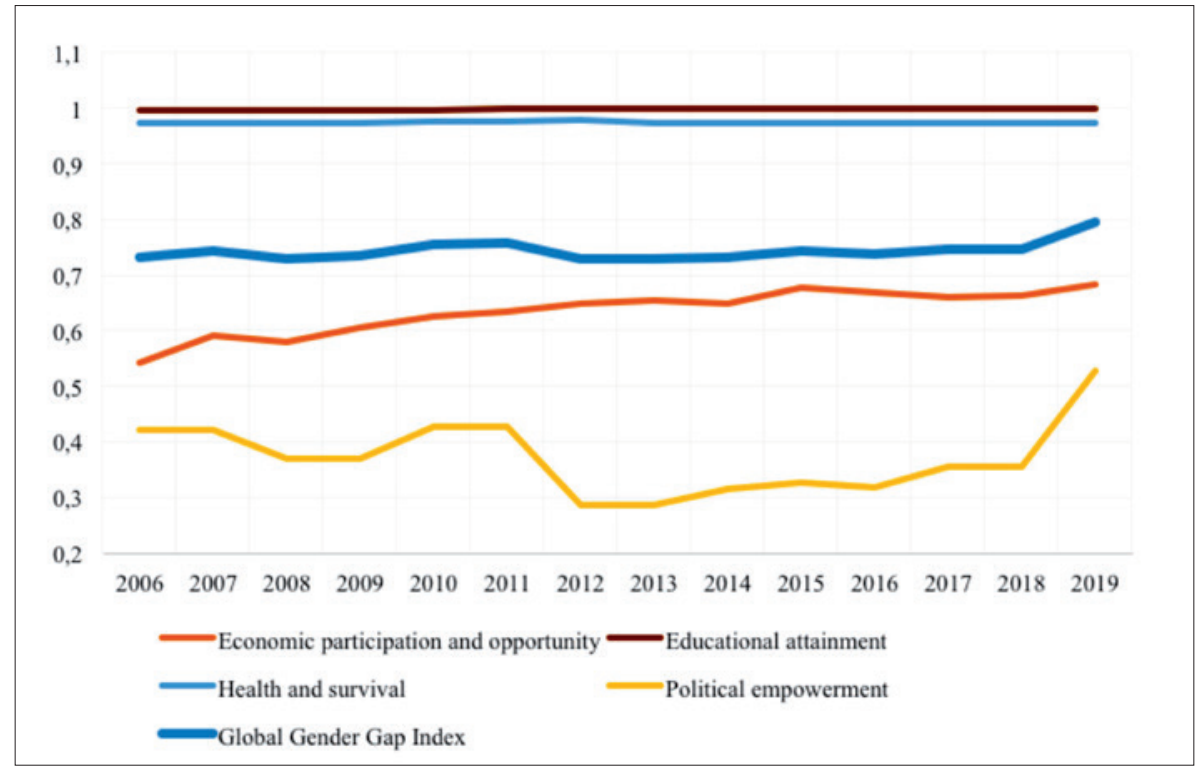

Fuente: elaboración propia a partir de datos del World Economic Forum.

Figura 2. Indicadores para España de empoderamiento político en el GGGR.

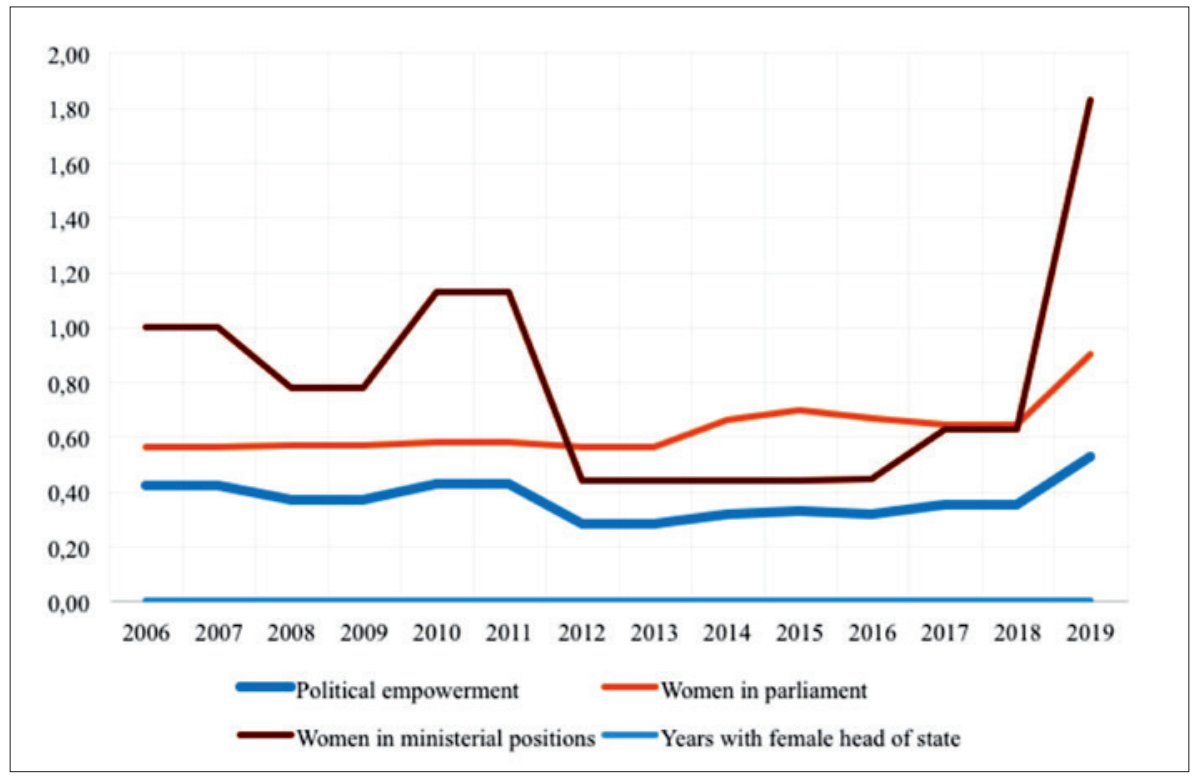

Fuente: elaboración propia a partir de datos del World Economic Forum.

Además, en la pasada legislatura, el gobierno socialista realizó una serie de avances en el ámbito de igualdad de género utilizando para ello la vía de urgencia. Así, el Real Decreto-ley 6/2019, de 1 de marzo, de medidas urgentes para garantía de la igualdad de trato y de oportunidades entre mujeres y hombres en el empleo y la ocupación, contiene 7 artículos que se corresponden con la modificación de siete normas con rango de ley que inciden de forma directa en la igualdad entre mujeres y hombres. Entre ellos, es de destacar la equiparación progresiva del 
permiso de paternidad con el de maternidad, otorgando a los padres hasta 16 semanas de permiso en 2021 para nacimientos y adopciones. El decreto tiene como objetivo reducir la representación insuficiente de las mujeres en el mercado laboral.

El gobierno también ha extendido la obligación de tener planes de igualdad de género a empresas con más de 50 empleados; antes el límite era empresas con 250 trabajadores. Estos planes deben incluir una auditoría salarial y medidas de corresponsabilidad y prevención del acoso sexual. El decreto también refuerza las políticas públicas destinadas a la atención y cuidados de menores, así como de las personas en situación de dependencia.

\section{Aspectos a mejorar para alcanzar la paridad de género en España}

A pesar de avanzar en todos los aspectos de la participación económica, existen áreas donde hay todavía mucho espacio de mejora para conseguir la paridad de género. Así, la participación de las mujeres en el mercado laboral todavía está por debajo de la de los hombres (68,8 \% frente a un 78,9 \% respectivamente) y aún quedan por cerrar grandes brechas en salarios, ingresos (según Eurostat, 2017, los ingresos brutos por hora de las mujeres fueron un 15,1\% inferiores a los de los hombres) y presencia de mujeres en puestos de gestión. Esto muestra que todavía existen fuertes barreras culturales y prácticas empresariales que limitan las oportunidades laborales de las mujeres.

De hecho, la participación de las mujeres en los consejos de administración de las empresas cotizadas sigue siendo baja (22 \%), no alcanzando el objetivo del $30 \%$ entre las empresas del IBEX 35 previsto para 2020. Las mujeres españolas siguen enfrentando el llamado «techo de cristal» para acceder a los órganos de gestión del sector privado.

Otro punto relacionado con la participación femenina en la economía es la educación. Uno de los mayores desafíos para los próximos años será prevenir la brecha económica de género relacionada con la infrarrepresentación de las mujeres en las profesiones emergentes (Tecnologías de la Información y Comunicaciones, Ingeniería, manufactura y construcción, Ciencias, Matemáticas y Estadística) (figura 3). Hoy las mujeres tienen una mayor representación en puestos que se están automatizando con condiciones de trabajo más pobres. Según la CEOE (2019), la mayoría de las mujeres españolas tienen carreras relacionadas con la educación, la salud y el bienes$\operatorname{tar}(>75 \%)$, mientras que los hombres predominan en carreras técnicas como la ingeniería (75\% - $87 \%$ o los relacionados con las TIC (90\%). Eliminar los obstáculos que impiden a las mujeres acceder a los sectores de la ciencia, la investigación y la tecnología será clave para modificar la orientación académica actual, que es vital para luchar contra nuevas formas de brecha de género. 
Figura 3. Indicador para España de educación y habilidades en el GGGR.

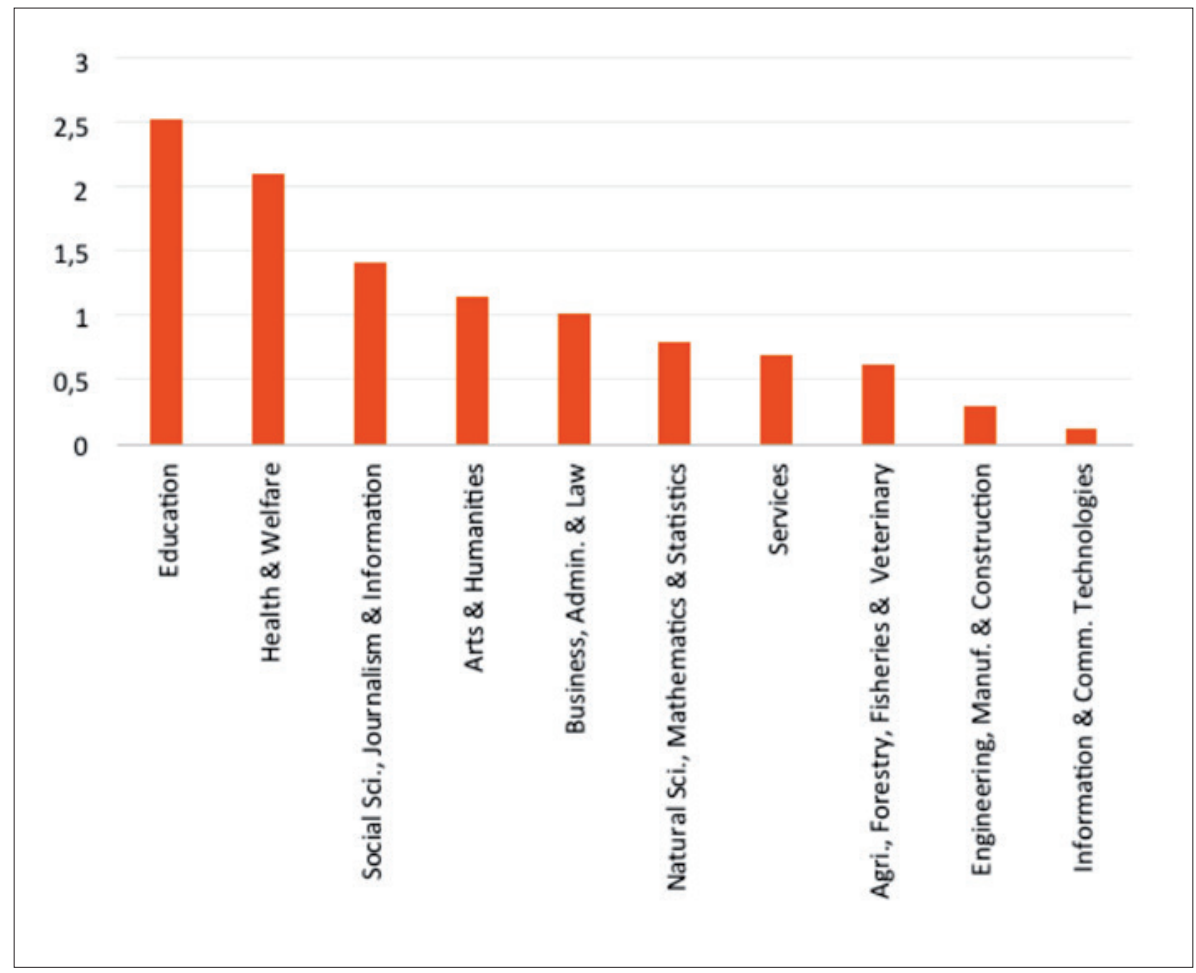

Fuente: elaboración propia a partir de datos del World Economic Forum (2020).

Así, los datos nos muestran que, a pesar de que las mujeres suponen casi el $60 \%$ de los egresados universitarios (MECD, 2018), menos del 30 \% de las mujeres (MECD, 2018) optan por carreras de ciencias, tecnología, ingeniería y matemáticas (esto es, las conocidas como STEM, acrónimo de las disciplinas académicas: Science Technology Engineering and Mathemathics). Se trata de un fenómeno global, ya que en casi todos los países de la OCDE menos de 1 de cada 3 graduados en ingeniería son mujeres (OCDE, 2016).

Lograr revertir estos datos y eliminar las barreras que alejan a las mujeres de la ciencia se trata de un reto complejo que debe abordarse desde un punto de vista amplio y multidisciplinar, reduciendo los estereotipos y prejuicios que todavía existen, visibilizando el talento femenino en estas disciplinas y garantizando una educación libre de sesgos de género. Esto será clave para modificar la orientación académica actual, que es vital para luchar contra nuevas formas de brecha de género.

\section{La cuota de género en los consejos de administración de las empresas españolas}

La Ley 3/2007, de 22 de marzo para la Igualdad Efectiva entre hombres y mujeres, introducía en su artículo 75 lo que se conoce como una cuota de género «blanda", que recomendaba que "las sociedades obligadas a presentar cuenta de pérdidas y ganancias no abreviada procurarán incluir en su consejo de administración un número de mujeres que permita alcanzar una presencia equilibrada de mujeres y hombres» hasta alcanzar el objetivo del 40 $\%$ en el 2015. El porcentaje de mujeres en los consejos de las 1.000 mayores empresas españolas solo ha crecido desde un 6,6 \% por ciento en 2005 hasta un 11,8 \% en 2014, bastante lejos del objetivo del $40 \%$ establecido. De hecho, tan solo un 4,6 \% de estas empresas cumple con el objetivo de paridad en sus consejos (Mateos de 
Cabo et al., 2019). Esta escasa eficacia de la ley para alcanzar su objetivo viene determinada, en buena medida, por la no inclusión de ningún tipo de sanciones para aquellas empresas que no cumplieran con el artículo 75.

El único incentivo positivo para cumplir con la cuota aparece en su artículo 34.2, donde se prevé que el gobierno podrá establecer una preferencia en la adjudicación de contratos públicos a aquellas empresas que cumplan con las directrices dadas en la ley para fomentar la igualdad efectiva entre mujeres y hombres en el mercado de trabajo.

En su estudio, Mateos de Cabo et al. (2019) obtienen tres resultados que son importantes para no repetir los mismos errores en futuras iniciativas políticas y/o legislativas:

La ley no ha tenido efectos significativos sobre el conjunto de grandes empresas españolas. De hecho, en el periodo transcurrido entre 2005 y 2014 tan solo se ha observado un pequeño crecimiento anual de 0,5 puntos porcentuales en la representación femenina en los consejos. Dicho crecimiento, además, no puede considerarse directamente atribuible a la ley, pues no hay un salto cuantitativo significativo tras su entrada en vigor. Parece que se trata más bien de un lento "crecimiento orgánico» con el que tendríamos que esperar alrededor de 60 años para observar paridad en los consejos.

Sí se ha observado, sin embargo, algún progreso entre aquellas empresas con dependencia de los contratos públicos (un crecimiento de 6 puntos porcentuales en representación femenina en los consejos y de 4 puntos en el porcentaje de empresas con consejos paritarios). Dicho avance puede ser atribuido al hecho de que las empresas con dependencia de ingresos públicos están directamente afectadas por el incentivo ofrecido en la ley de dar preferencia en la adjudicación de contratos públicos a quienes cumplan con el objetivo de paridad en sus consejos. Este resultado demuestra la efectividad de las acciones afirmativas de género (bien en forma de incentivos y/o de sanciones) y la necesidad de hacerlas extensivas a todas las grandes empresas y no solo a un pequeño porcentaje de las mismas, si se quiere conseguir en un espacio temporal razonable, la equidad de género en los consejos.

Por último, el cumplimento de la cuota por parte de las empresas no ha venido acompañado de un aumento de los ingresos procedentes de los contratos públicos. Este hecho puede poner en peligro el pequeño avance ya logrado. Y es que para que un incentivo sea eficaz, este tiene que ser creíble y aplicarse de manera efectiva, de no ser así, se corre el riesgo de que las empresas den marcha atrás al no percibir un compromiso claro e inequívoco del gobierno en relación con el cumplimiento de la cuota.

La situación legislativa actual con respecto a las cuotas de género parece más bien estancada. En el mes de septiembre del 2018 el Grupo Socialista registró en el Congreso de los Diputados la Proposición de Ley 122/000268 para garantizar la igualdad de trato y de oportunidades entre mujeres y hombres en el empleo y la ocupación, que establece que «la composición del consejo de administración de las sociedades obligadas a presentar cuenta de pérdidas y ganancias deberá cumplir con el principio de presencia equilibrada a partir del año 2023».

Al acabar la XII legislatura todas las proposiciones de ley que no habían sido aprobadas caducaron, por lo que en esta nueva legislatura sería necesario volver a iniciar todo el proceso y de momento no se ha presentado ninguna proposición de ley al respecto. Antes de que caducara, el Consejo de Ministros aprobó el Real Decretoley 6/2019, de 1 de marzo, de medidas urgentes para garantizar la igualdad de trato y de oportunidades entre mujeres y hombres en el empleo y la ocupación, que recogía parte de las medidas de la proposición de ley, pero no la parte relativa a las cuotas en los consejos al ser de difícil justificación incluirla en el Real Decreto como medida urgente.

Esta proposición de ley hubiera supuesto un cierto avance en la igualdad de género en los consejos de las empresas españolas con respecto a la anterior ley, pues su redacción supone pasar del «procurarán» que establecía la del 2007, a un grado mayor de coercitividad al incluir el término «deberá» en su redacción.

Recientemente, en enero de 2020, la CNMV ha anunciado que modificará el Código de Buen Gobierno para que las mujeres representen, al menos, el 40 \% (más en línea con la Ley de lgualdad del 2007) de los consejos de las sociedades cotizadas. Ello aumenta en 10 puntos porcentuales el peso sobre el Código de Buen Gobierno del 2015. Además, a diferencia de lo que hacía el código anterior, para cumplir la recomendación ya no bastará con decir que se tiene una política encaminada a incentivar la diversidad, sino que las empresas deberán explicar por qué no la cumplen (principio conocido como «cumplir o explicar»). 


\section{Conclusión y propuestas de mejora}

Como conclusión, podemos decir que España ha realizado un gran avance en el ámbito de la igualdad de género hasta situarse entre los 10 mejores países en términos de igualdad según el Foro Económico Mundial. Esta mejora ha sido posible gracias al empoderamiento político de las mujeres que ha traído consigo el cambio de gobierno y a algunas medidas complementarias relacionadas con el mercado laboral, como la progresiva igualación de permisos de paternidad y la obligación de realizar planes de igualdad de género las empresas de más de 50 empleados. No obstante, todavía queda mucho espacio de mejora para que estos avances no dependan de los avatares políticos, especialmente en lo que se refiere al empoderamiento económico de las mujeres.

En este sentido, un aspecto sobre el que habrá que trabajar será reforzar la presión política y/o reguladora para conseguir avanzar hacia la paridad en los consejos y altos puestos directivos de las empresas españolas en un plazo razonable de tiempo. Para ello, y a la vista del fracaso que supuso el intento de la Ley de Igualdad de 2007 a este respecto sería necesario, en primer lugar, conseguir un compromiso político institucional. De hecho, se abren posibilidades de que vuelvan a la agenda política desarrollos legislativos en torno a la cuota en los consejos, o al menos, el planteamiento de una amenaza de cuota dura. La presión política y/o regulatoria debería ir acompañada de otras medidas regulatorias complementarias, en particular las que provienen de políticas públicas que fomentan mayores permisos de paternidad y una mayor corresponsabilidad en tareas domésticas y cuidado de dependientes. Resulta también necesario profesionalizar los procesos de selección para garantizar que sean imparciales y que la mejor combinación de talentos llegue a los consejos. Esto podría conducir a un código de conducta que establezca el compromiso entre las empresas de búsqueda (headhunters), para incluir a más mujeres entre sus candidatos. La creación de un business case (o justificación económica) para España ayudaría a que las empresas españolas apreciaran las ventajas de lograr que las mujeres formen parte de un grupo de talentos amplio y diverso. Y finalmente, también sería deseable un esfuerzo más coordinado de los defensores de la igualdad de género, con la participación de agentes políticos, empresas y líderes visibles (mujeres y hombres) que puedan mostrar los beneficios de crear equipos diversos a la hora de mejorar los procesos de toma de decisiones.

Otro aspecto clave que no conviene olvidar para incrementar el empoderamiento económico de las mujeres es la transformación digital que se está produciendo en el mercado laboral. Es este un problema no menor, ya que dada la actual segregación de hombres y mujeres en diferentes sectores económicos y la reducida proporción de mujeres que optan por carreras STEM, puede contribuir a acentuar las brechas actuales del mercado laboral, ya que se prevé que la demanda de perfiles STEM aumentará. Por ello, resulta necesario intensificar las acciones dedicadas a fomentar las vocaciones en estos campos entre las mujeres. En este sentido, el Foro Económico Mundial indica una serie de estrategias que ayudarían a cerrar estas brechas. Primero, se señala una necesidad urgente de aumentar la oferta y visibilidad de mujeres con habilidades técnicas disruptivas. Para asegurar que las profesiones del futuro puedan alcanzar la paridad de género en las próximas décadas, es necesario enfocarse en realizar un esfuerzo de actualización y mejora de las habilidades de aquellas mujeres que ya se encuentren en el mercado laboral o buscando reincorporarse después de un periodo de actividad y estén interesadas en expandir su rango de capacidades. Al mismo tiempo, las empresas deberían diseñar una agenda de diversidad e inclusión rigurosa que dirigiera las prácticas de selección y reclutamiento para hacer pleno uso del pool femenino de talento disponible y asegurar un lugar de trabajo más inclusivo que ayude a retener y desarrollar a las mujeres actualmente empleadas en profesiones de frontera científico-tecnológica. Por último, merece destacar el importante papel que juega la educación en la eliminación de roles y estereotipos de género que pueden alejar a las niñas de estas profesiones. En este sentido, intervenciones como talleres y campamentos de ciencia y tecnología, charlas, eventos, reuniones y debates con mujeres protagonistas en el ámbito científico y tecnológico se han mostrado especialmente eficaces para reducir la imagen estereotipada del genio científico con capacidades innatas, sin vida personal y cuyo trabajo tiene pocas o nulas implicaciones prácticas en el mundo real (González et al., 2020). 


\section{Bibliografía}

CEOE (2019). Análisis de la brecha salarial de género en España. Identificando las causas para conocer las soluciones. www.pwc.es/es/publicaciones/diversidad/analisis-brecha-salarial-genero-espana-ceos-pwc.pdf [consultado el 23 de enero de 2020].

EUROSTAT (2017). Gender Pay Gap Statistics. https://ec.europa.eu/eurostat/statistics-explained/index. php?title=Gender_pay_gap_statistics [consultado el 23 de enero de 2020].

GONZÁLEZ, S.; MATEOS de CABO, R.; SÁINZ, M. (2020). «Girls in STEM: Is it a role model thing?» Mimeo. https://doi.org/10.2139/ssrn.3541939.

MATEOS de CABO, R.; TERJESEN, S.; ESCOT, L.; GIMENO, R. (2019). «Do 'soft law' board gender quotas work? Evidence from a natural experiment». European Management Journal. Vol. 37, núm. 5, pág. 611-624. https://doi.org/10.1016/j.emj.2019.01.004.

Ministerio de Educación y Ciencia (2018). Estadística de estudiantes. Curso 2017/2018. [Consultado el 21 de enero de 2020]. Disponible en: www.mecd.gob.es/servicios-al-ciudadano-mecd/estadisticas/educacion/universitaria/estadisticas/alumnado/2017-2018_Rend.html

OCDE. 2016. Education and Training. Organización para la Cooperación y Desarrollo Económico [consultado el 21 de enero de 2020]. Disponible en: https:/stats.oecd.org/Index.aspx?=DatasetCode=RGRADSTY.

Proposición de Ley para garantizar la igualdad de trato y de oportunidades entre mujeres y hombres en el empleo y la ocupación. (122/000268)

Real Decreto-ley 6/2019, de 1 de marzo, de medidas urgentes para garantía de la igualdad de trato y de oportunidades entre mujeres y hombres en el empleo y la ocupación.

WORLD ECONOMIC FORUM (2020). Global Gender Gap Report 2020. World Economic Forum.

Cita recomendada: GONZÁLEZ, Susana; MATEOS, Ruth. Balance y propuestas de mejora. La (des)igualdad de género en las organizaciones: avances y estancamientos en España. Oikonomics [en línea]. Mayo 2020, n. 13, pp. 1-9. ISSN: 2339-9546. DOI: https://doi.org/10.7238/o.n13.2004 


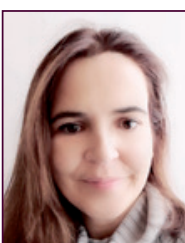

\section{Susana González}

Profesora colaboradora del departamento de Economía de la Empresa de la Universidad CEU San Pablo. Miembro de la línea de investigación de Liderazgo Femenino de la Cátedra USPCEU-Mutua Madrileña.

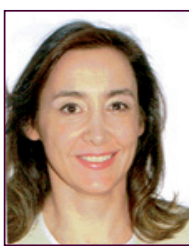

\section{Ruth Mateos}

Profesora titular del departamento de Economía de la Empresa de la Universidad CEU San Pablo. Directora de la línea de investigación de Liderazgo Femenino de la Cátedra USPCEU-Mutua Madrileña. Actualmente es la investigadora principal de un proyecto del Plan Nacional de I+D+i sobre Mujeres y Liderazgo Empresarial financiado por el Ministerio de Economía.

Los textos publicados en esta revista están sujetos -si no se indica lo contrario- a una licencia de Reconocimiento 4.0 Internacional de Creative Commons. Puede copiarlos, distribuirlos, comunicarlos públicamente, hacer obras derivadas siempre que reconozca los créditos de las obras (autoría, nombre de la revista, institución editora) de la manera especificada por los autores o por la revista. La licencia completa se puede consultar en https://creativecommons.org/licenses/by/4.0/deed.es_ES.

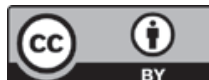

